4. Cordioli AV, Heldt E, Bochi DB, Margis R, Souza MB, Tonello J, Teruchkin B, Kapczinski F. Cognitive-behavioral group therapy in obsessive-compulsive disorder: a clinical trial. Rev Bras Psiquiatr. 2002;24(3):113-20.

5. Shavitt RG, Belotto C, Curi M, Hounie AG, Rosario-Campos MC, Diniz JB, Ferrao YA, Pato MT, Miguel EC. Clinical features associated with treatment response in obsessive-compulsive disorder. Compr Psychiatry. 2006;47(4):276-81.
Leonardo F Fontenelle Programa de Ansiedade e Depressão, Departamento de Psiquiatria e Medicina Legal, Instituto de Psiquiatria, Universidade Federal do Rio de Janeiro (IPUB/UFRJ),

Rio de Janeiro (RJ), Brasil

Mauro V Mendlowicz Departamento de Psiquiatria e Saúde Mental, Instituto de Saúde da Comunidade, Universidade Federal Fluminense (UFF), Niterói (RJ), Brasil

\section{São as compulsões de checagem e lavagem marcadores válidos de diferentes subtipos do TOC?} Are checking and washing compulsions
valid markers of different subtypes of OCD?

\section{Sr. Editor,}

Agradecemos pelo interesse em nosso estudo ${ }^{1}$ e pela oportunidade de esclarecer alguns pontos. Em primeiro lugar, o estudo em questão resulta de uma análise secundária de dados provenientes da amostra de pacientes com transtorno obsessivo-compulsivo (TOC) atualmente em acompanhamento no Programa de Ansiedade e Depressão do Instituto de Psiquiatria da Universidade Federal do Rio de Janeiro (IPUB/UFRJ). Todos os indivíduos recrutados para nosso estudo foram selecionados de acordo com os critérios de inclusão e exclusão descritos em sua seção de métodos. No entanto, como em todos os estudos conduzidos com amostras clínicas de pacientes com TOC, há uma possível carência de pacientes com colecionismo patológico, já que estes indivíduos apresentam menores níveis de insight ${ }^{2}$ e de adesão ao tratamento. ${ }^{3}$ Em um estudo prévio de nossa autoria, somente $15,6 \%$ dos pacientes com TOC apresentavam colecionismo patológico como um dos principais sintomas. ${ }^{4}$

Gropo et al. nos indagam a respeito da história familiar e da resposta terapêutica dos subtipos de pacientes por nós estudados. No entanto, acreditamos que somente investigações sistemáticas, envolvendo entrevistas clínicas de familiares de pacientes com TOC com diferentes fenótipos e controles saudáveis, são capazes de fornecer informações suficientemente confiáveis para validações de subtipos clínicos e biológicos de diferentes transtornos psiquiátricos. Infelizmente, tal tipo de avaliação encontrava-se fora do escopo de nossa investigação. De maneira semeIhante, estudos que envolvem acompanhamento clínico naturalístico são sujeitos a inúmeras variações não controláveis do ponto de vista metodológico, o que torna esta estratégia inadequada para estudos que visam validar subtipos do TOC. Portanto, ainda que tais sugestões fossem por nós absorvidas, a mera descrição destes achados, como sugerido por Gropo et al., não contribuiria de maneira significativa para a validação dos fenótipos aqui descritos.

Estudos futuros, com metodologia adequada, devem incorporar não apenas história familiar e resposta ao tratamento, mas também achados neuroquímicos, neuroanatômicos e neuroimunológicos, no intuito de confirmar ou refutar a existência de subtipos do TOC baseados em compulsões de verificação e lavagem.

\section{Referências}

1. Fontenelle LF, Mendlowicz MV, Versiani M. Clinical subtypes of obsessive-compulsive disorder based on the presence of checking and washing compulsions. Rev Bras Psiquiatr. 2005;27(3):201-7.

2. Greenberg D. Compulsive hoarding. Am J Psychother. 1987;41(3):409-16.

3. Mataix-Cols D, Marks IM, Greist JH, Kobak KA, Baer L. Obsessivecompulsive symptom dimensions as predictors of compliance with and response to behaviour therapy: results from a controlled trial. Psychother Psychosom. 2002;71(5):255-62.

4. Fontenelle LF, Mendlowicz MV, Soares ID, Versiani M. Patients with obsessive-compulsive disorder and hoarding symptoms: a distinctive clinical subtype? Compr Psychiatry. 2004;45(5):375-83. 